\title{
Dietary methyl content regulates opioid responses in mice
}

\author{
This article was published in the following Dove Press journal: \\ Journal of Pain Research \\ 27 March 2013 \\ Number of times this article has been viewed
}

\author{
De-Yong Liang ${ }^{1,2}$ \\ Yuan Sun ${ }^{1,2}$ \\ J David Clark ${ }^{1,2}$ \\ 'Department of Anesthesiology, \\ Veterans Affairs Palo Alto Health Care \\ System, Palo Alto, ${ }^{2}$ Stanford University \\ School of Medicine, Stanford, CA, USA
}

Correspondence: De-Yong Liang Department of Anesthesiology II2A, Veterans Affairs Palo Alto Health Care System, 380I Miranda Ave, Palo Alto, CA 94304, USA

Tel +I 6504935000 ext 68838

$\mathrm{Fax}+\mathrm{I} 6508523423$

Email dyliang@stanford.edu
Background: Large interindividual differences in clinical responses to opioids and the variable susceptibility to abuse of this class of drugs make their use problematic. We lack a full understanding of the factors responsible for these differences. Dietary factors including methyl donor content have been noted to alter multiple physiological and behavioral characteristics of laboratory animals. The purpose of this research was to determine the effects of dietary methyl donor content on opioid responses in mice.

Methods: Groups of male C57BL/6J mice were treated with high and low methyl donor diets either in the perinatal period or after weaning. Analgesic responses to morphine, as well as tolerance, opioid-induced hyperalgesia, and physical dependence were assessed.

Results: Mice fed high and low methyl donor diets showed equal weight gain over the course of the experiments. Exposure to a high methyl donor diet in the perinatal period enhanced physical dependence. Dietary methyl donor content also altered analgesic responses to low doses of morphine when the dietary treatments were given to the mice after weaning. Opioid-induced hyperalgesia was unaltered by dietary methyl donor content.

Conclusion: High and low methyl donor diet treatment has selective effects on opioid responses depending on the timing of exposure. These findings suggest that examination of DNA methylation patterns in specific brain regions linked to opioid analgesia and dependence may provide specific explanations for dietary effects on opioid responses.

Keywords: opioid, methylation, tolerance, hyperalgesia, dependence

\section{Introduction}

Opioids are the cornerstone of management for severe pain, yet large interindividual responses are seen clinically and the abuse potential of this class of drugs makes their use problematic. The reasons for these differences are poorly understood. Data derived from both humans and animal models suggest that components of an individual's diet may alter responses to opioids including analgesia, physical dependence, and abuse. The consumption of sweet and fatty foods, for example, predisposes humans and animals to addictive behaviors. ${ }^{1}$ Cholesterol-rich diets tend to potentiate opioid analgesic effects in mice and possibly in humans. ${ }^{2}$ On the other hand, dietary restriction of alpha linolenic acid failed to alter opioid analgesia in mice, although it did change the locomotor response. ${ }^{3}$ Looking more specifically at dietary effects on opioid pathways, a high-fat diet altered $\mu$-opioid receptor expression in several central nervous system centers suggested to support binge eating disorders. ${ }^{4}$

Strong effects of diet in early life on opioid responses and gene expression have been noted. When rat pups were exposed to an undernourishment protocol, they 
displayed less opioid withdrawal when subsequently treated with morphine followed by administration of an opioid receptor antagonist. ${ }^{5}$ In other experiments using pregnant mice, a high-fat maternal diet increased expression of the $\mu$-opioid receptor and other addiction-related genes in the brains of the pups. The mechanism for these differences in gene expression involved differences in methylation of the promoter regions of the differentially expressed genes. ${ }^{6}$ Altered patterns of DNA methylation in response to dietary differences in methyl donor or fat content are believed to explain a number of additional effects in animals and humans. Very recently, investigators found that the methylation pattern was altered at 82 distinct genomic loci when mice were exposed perinatally to a high versus low methyl donor diet. ${ }^{7}$

In light of the results of the aforementioned studies, we hypothesized that exposing mice to high versus low methyl donor diets in the perinatal period would alter opioid analgesia, tolerance, dependence and opioid-induced hyperalgesia later in life.

\section{Materials and methods}

\section{Animal subjects}

All the animal experiments were done with the approval of the Veterans Affairs Palo Alto Health Care System Institutional Animal Care and Use Committee and complied with the Guide for the Care and Use of Laboratory Animals available through the National Academy of Sciences. The mice were housed under pathogen-free conditions with soft bedding, and were provided food and water ad libitum with a 12:12 light:dark cycle.

\section{Methyl donor diet and breeding protocol}

High and low methyl donor chows were purchased from Research Diets (New Brunswick, NJ, USA). High methyl supplementation was added using folic acid, vitamin B12, choline, L-methionine, zinc, betaine, and genistein. All these methyl donors are involved in methyl metabolism. ${ }^{8}$ The difference in ingredients in the low and high methyl donor diets is summarized in Table 1. Each of the experimental diets supported normal growth and was not associated with any known dietary deficiency syndromes. On completion of testing, each animal had been exposed to either the high or low methyl donor diet for approximately 6 weeks either in the perinatal period or later in life.

For the perinatal methyl donor supplementation studies, male and female $\mathrm{C} 57 \mathrm{BL} / 6 \mathrm{~J}$ mice were purchased from
Table I Methylation-related components of high methyl and low methyl donor diets*

\begin{tabular}{lll}
\hline Ingredient & $\begin{array}{l}\text { Low methylation } \\
\mathbf{( g ,}, \mathbf{)}\end{array}$ & $\begin{array}{l}\text { High methylation } \\
\mathbf{( g ,}, \mathbf{)}\end{array}$ \\
\hline L-methionine & 0 & 7.5 \\
Soybean oil & 0 & 70 \\
Corn oil & 70 & 0 \\
Zinc carbonate, 52.1\% zinc & 0 & 0.288 \\
Folic acid & 0 & 0.015 \\
Betaine & 0 & 15 \\
Cyanocobalamin, 0.1\% & 0 & 0.15 \\
Choline bitartrate & 2.5 & 36.6 \\
Genistein & 0 & 0.3
\end{tabular}

Note: *Formulated by Research Diets Inc, New Brunswick, NJ, USA.

Jackson Laboratories (Bar Harbor, ME, USA) at 5 weeks of age. Twelve females were randomly divided into two groups to receive high or low methyl donor diets. The diets were provided for 2 weeks prior to mating and throughout gestation and lactation. ${ }^{7,9,10}$ At postnatal day 21, the pups were weaned and received the standard rodent diet (2018, Harlan-Teklad, Madison, WI, USA). This diet was continued during the experiments, which began at 8 weeks of age.

For the postweaning methyl donor supplementation studies, C57BL/6J dams with postnatal day 14 pups were purchased from Jackson Laboratories. These mice received a standard rodent diet after arrival at our institution. At postnatal day 21 , the pups were weaned and randomized to receive either high or low methyl donor diets. The diets were provided until they were used in behavioral tests at 8 weeks of age, and continued through the testing period.

\section{Mechanical nociceptive thresholds}

Mechanical allodynia was assessed using nylon von Frey filaments according to the "up-down" algorithm previously described by Chaplan et al. ${ }^{11}$ For these measurements, mice were placed on mesh platforms within transparent plastic cylinders. After 15 minutes of acclimation, nylon fibers of sequentially increasing stiffness (0.2-2 g, seven fibers) were applied to the plantar surface of one hind paw, and left in place for 5 seconds. Withdrawal of the hind paw from the fiber was scored as a response. If no response was observed, the next stiffer fiber was applied to the same paw; if a response was observed, a less stiff fiber was applied. Testing continued until four fibers had been applied after the first withdrawal response, allowing estimation of the mechanical withdrawal threshold. The monofilament fibers were purchased from Stoelting (Wood Dale, IL, USA). This data-fitting algorithm allowed the use of parametric statistics for analysis. 


\section{Thermal nociceptive thresholds}

Paw withdrawal response latencies to noxious heat stimulation were measured using the method of Hargreaves et al as modified for use in mice. ${ }^{12}$ In this assay, the mice were placed on a temperature-controlled glass platform $\left(29^{\circ} \mathrm{C}\right)$ in a clear plastic enclosure similar to that described above. After 30 minutes of acclimatization, a beam of focused light was directed towards the same area of the hindpaw as described for the von Frey assay. A 20 -second cutoff time of paw withdrawal was used to prevent tissue damage. In these experiments, the light beam intensity was adjusted to provide approximately 10 seconds of baseline latency in the control mice. Three measurements were made per animal per test session separated by at least one minute.

\section{Morphine dose-response}

Cumulative morphine dose-response curves were constructed using the tail-flick assay and methods similar to those we have described previously. ${ }^{13}$ For these measurements, the mice were gently restrained within a cone-shaped tube made of cotton toweling. Using a tail-flick analgesic apparatus (Columbus Instruments, Columbus, OH, USA), tail-flick latency was measured with 0.1 -second precision. A 10 -second cutoff time was used to prevent tissue sensitization or damage. Two measurements were made per mouse with the light beam focused on two different points $1 \mathrm{~cm}$ apart on the tail. The lamp intensity was identical for all animals, providing baseline tail-flick measurements of 3-4 seconds. For assessment of tolerance, these dose-response experiments followed 18 hours after the final dose of morphine was given as part of the chronic morphine administration protocol. The cumulative doses of morphine used were 1,3 , and $10 \mathrm{mg} / \mathrm{kg}$. Tail-flick latency was determined 25 minutes after morphine injection, as established in preliminary experiments, in which 25 minutes is the time at which peak morphine effect was achieved. The percent maximal possible effect (\%) was determined using the following formula:

Percent maximal possible effect (\%) $=100 *$ (measured latency - baseline latency $) /$ (cutoff latency - baseline latency)

\section{Chronic morphine administration}

After baseline nociceptive testing, morphine (Sigma-Aldrich, St Louis, MO, USA) was subcutaneously administered to the mice at $10 \mathrm{mg} / \mathrm{kg}$ twice per day on day $1,20 \mathrm{mg} / \mathrm{kg}$ twice per day on days $2-3$, and $40 \mathrm{mg} / \mathrm{kg}$ twice per day on day 4 , in $50-100 \mu \mathrm{L}$ volumes of $0.9 \% \mathrm{NaCl}^{13}$

\section{Precipitated withdrawal}

For determinations of physical dependence, the mice undergoing chronic morphine administrations were challenged with a subcutaneous injection of naloxone (Sigma Chemicals) $10 \mathrm{mg} / \mathrm{kg}$ in $100 \mu \mathrm{L}$ of saline, as described previously by our laboratory and others. ${ }^{14,15}$ After administration of naloxone, the mice were placed in transparent plastic cylinders, and the number of jumps during the following 15 minutes was counted.

\section{Statistical analysis}

All data are expressed as the mean \pm standard error of the mean, unless otherwise noted. Data for mechanical sensitivity, thermal sensitivity, tail-flick response, and dependence were analyzed by two-way analysis of variance followed by the Bonferroni post hoc test for multiple comparisons. Simple comparisons of two groups involved unpaired $t$-testing with two-tailed $P$ values. $P<0.05$ was considered to be statistically significant.

\section{Results Mice fed high or low methyl donor diets displayed equal weight gain}

Body weights at the time of experimentation were compared for the groups treated with high and low methyl donor diets. Although both diets were nutritionally complete, we sought to determine if any significant differences in growth rates would be present. For both methyl donor treatment protocols (perinatal and postweaning), the mice reached the same weights as shown in Figure 1. The average weights of the perinatal animals were $25.88 \mathrm{~g}$ and $25.99 \mathrm{~g}$ in the groups treated with high and low methyl donor diets, respectively $(P>0.05)$, and the average weights of postweaning animals were $23.25 \mathrm{~g}$ and $22.88 \mathrm{~g}$, respectively $(P>0.05)$. No gross developmental or motor differences were observed.

\section{High versus low perinatal methyl donor diets caused selective differences in opioid response}

We surveyed a group of clinically relevant opioid response phenotypes in mice exposed to high versus low methyl donor diets in the perinatal period. The mice were then tested at 8 weeks of age, approximately 5 weeks after beginning 


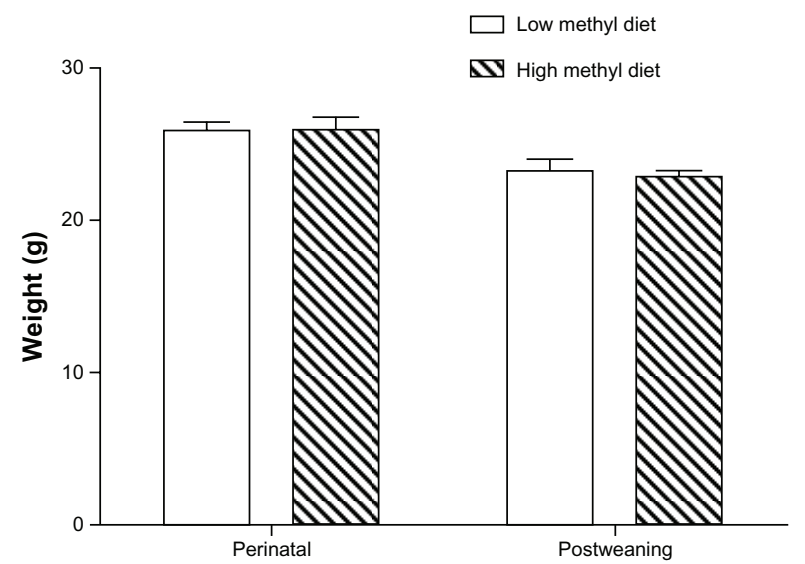

Figure I Growth patterns for male C57BL/6] mice fed high versus low methyl donor diets (eight mice per group).

Note: The weights of the mice were determined at 8 weeks of age when behavioral testing began.

a standard laboratory diet. First, we compared baseline mechanical and thermal nociceptive thresholds, as shown in Figure $2 \mathrm{~A}$ and $\mathrm{B}$. The mechanical thresholds at baseline were $1.001 \mathrm{~g}$ and $1.093 \mathrm{~g}$, respectively, in the high and low methyl donor diet groups, and thermal nociceptive thresholds were

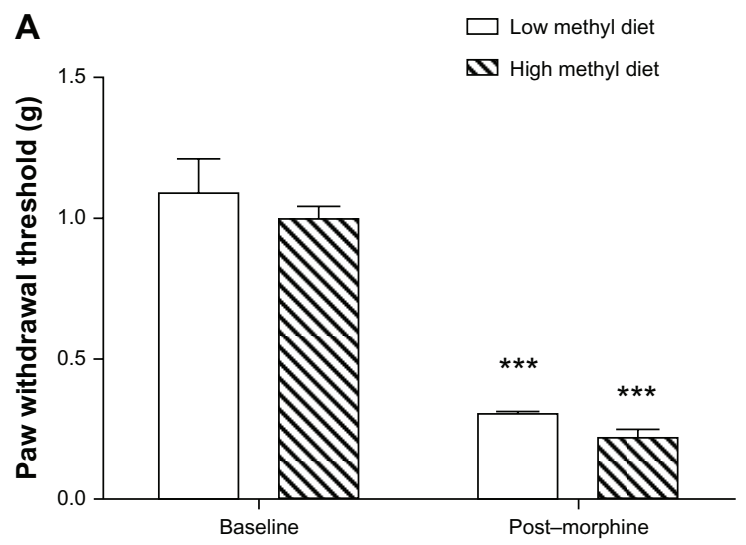

C

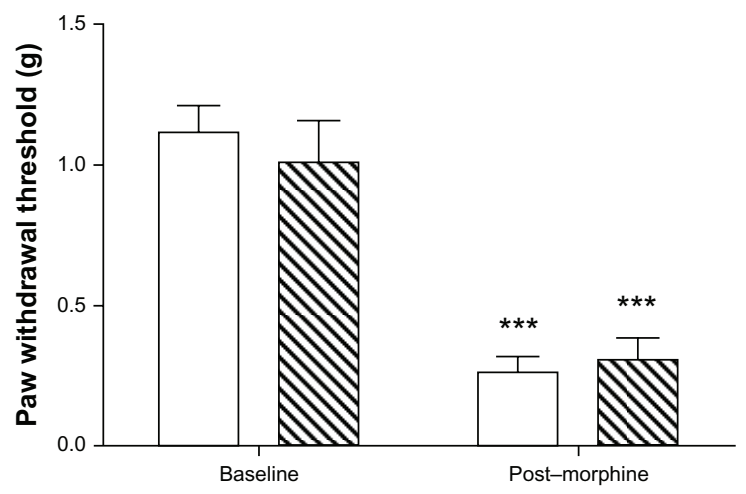

11.18 seconds and 11.68 seconds, respectively. These did not differ between the different dietary groups $(P>0.05)$, although the high methyl donor diet trended towards enhanced sensitivity. After completing a 4-day ascending morphine treatment protocol, used in several previous studies, ${ }^{13}$ the mechanical thresholds dramatically decreased to $0.2238 \mathrm{~g}$ and $0.3075 \mathrm{~g}$, respectively, in the high and low methyl donor diet groups (F1, $28=134.42, P<0.0001)$, and thermal thresholds were significantly reduced to 9.651 seconds and 9.739 seconds $(\mathrm{F} 1,28=11.91, P=0.0018)$. However, the mechanical and thermal sensitivity induced by morphine treatment did not show significant differences between the dietary groups, as shown in Figure 2A and B.

The analgesic effects of morphine were compared between groups of mice receiving high versus low methyl donor diets in the perinatal period. Across the range of morphine doses used, acute opioid administration induced dose-response analgesic efficacies obtained using the tail-flick test before chronic morphine treatment, but these were not significantly different between the high and low methyl donor diet groups $(P>0.05)$ as shown in Figure 3A. When the dose-response

B

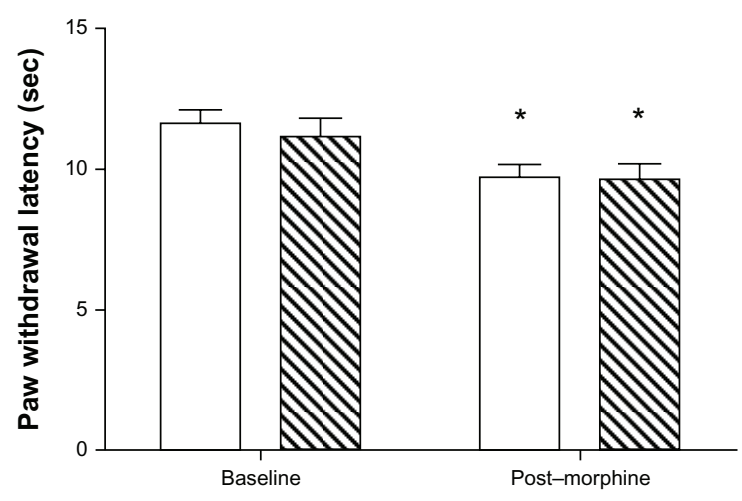

D

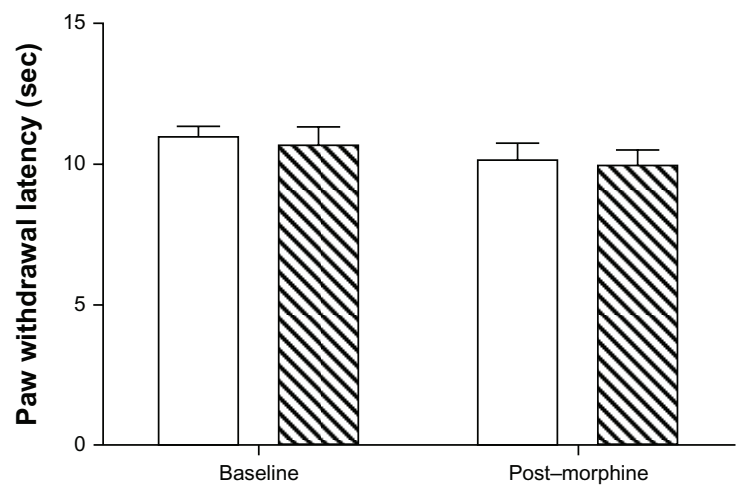

Figure 2 Mechanical and thermal nociceptive withdrawal thresholds for mice fed high versus low methyl donor diets (eight mice per group). (A and B) show the mechanical and thermal thresholds for mice receiving dietary treatment in the perinatal period. (C and $\mathbf{D})$ show the same data for mice receiving dietary treatment after weaning. Notes: $* P<0.05 ; * * * P<0.001$ for post-morphine versus baseline data. 

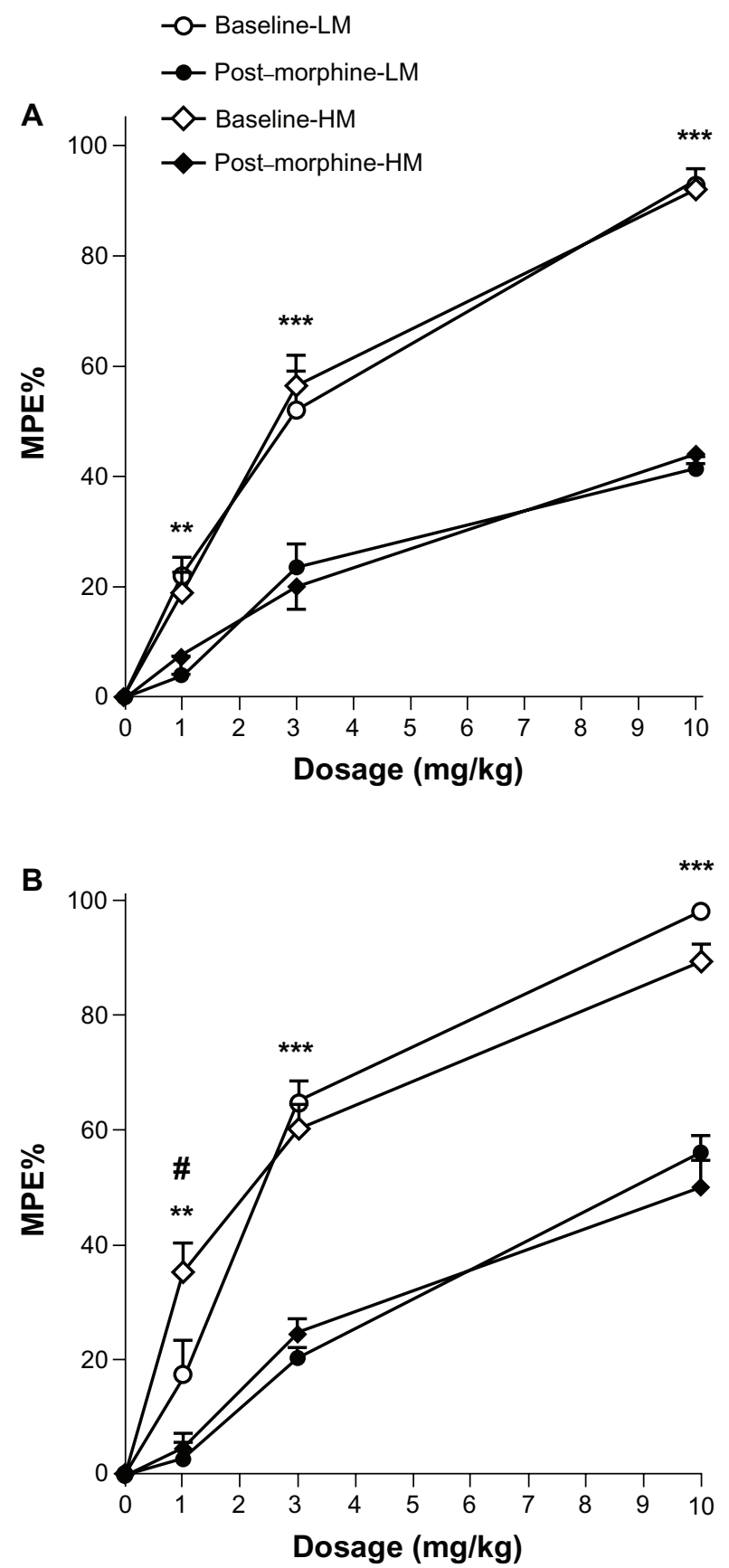

Figure 3 Morphine dose-response and tolerance in mice fed high versus low methyl donor diets. The analgesic effects of several doses of morphine were measured in groups of mice receiving different dietary treatments (eight mice per group). Measurements were done at baseline and following 4 days of escalating morphine treatment. (A) shows data from mice receiving dietary treatment in the perinatal period and (B) shows data from mice receiving different diets after weaning.

Notes: $* * P<0.01$; $* * * P<0.001$ for baseline versus post-morphine; ${ }^{*} P<0.05$ for high versus low methyl donor diet groups.

Abbreviations: LM, low methyl donor diet; HM, high methyl donor diet.

relationships were assessed after completing the 4-day ascending morphine treatment protocol, significant tolerance was observed as expected, but the degree of tolerance was indistinguishable between the dietary groups $(P>0.05$ for all doses, Figure 3A).
We next assessed physical dependence on morphine using naloxone-precipitated withdrawal. As shown in Figure 4, mice exposed perinatally to a high methyl donor diet showed significantly more jumping behavior than the low methyl donor-treated animals $(P<0.05)$. The jumping frequencies were 125.1 and 81.5 per 15 minutes, respectively, in the high and low methyl donor diet groups.

\section{High versus low methyl donor diets after weaning caused selective differences in opioid response}

The same battery of behavioral testing described for the perinatally treated mice was used on the mice starting on methyl donor altered dietary treatment after weaning. In this case, the switch from standard to supplemented or deficient chow was made 5 weeks before experimentation, and the mice received the modified diet throughout the period of testing. Again, no statistically significant differences were noted in baseline nociceptive sensitivity to thermal and mechanical stimuli (Figure 2C and D). The mechanical thresholds at baseline were $1.004 \mathrm{~g}$ and $1.111 \mathrm{~g}$, respectively, in the high and low methyl donor diet groups, and thermal nociceptive thresholds were 10.95 seconds and 10.70 seconds, respectively. Likewise, no differences were noted in thermal or mechanical responses after 4 days of morphine administration between the high and low methyl donor diet treatment groups $(P>0.05)$, although chronic morphine treatment induced significant mechanical allodynia, but not thermal hyperalgesia.

However, we did measure a significant difference between dietary groups in analgesic response to the lowest

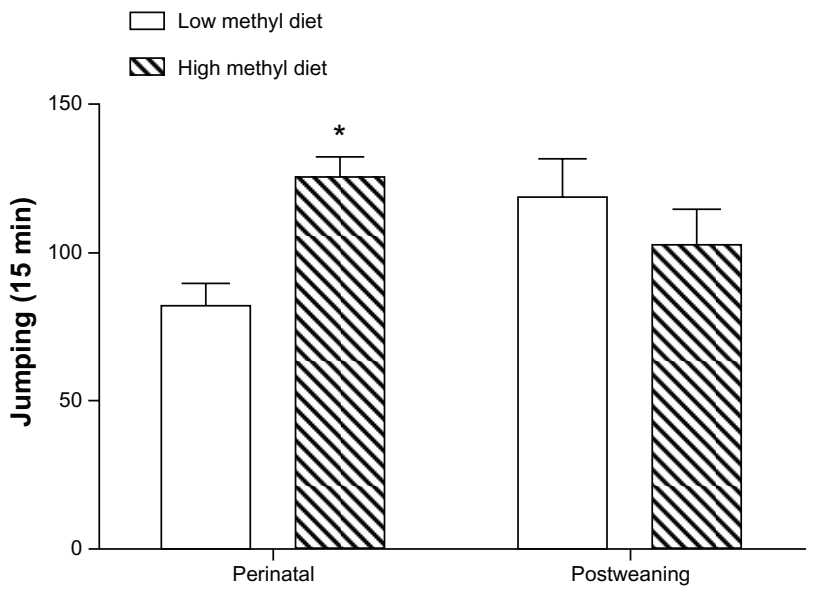

Figure 4 Physical dependence on morphine in mice fed high versus low methyl donor diets.

Notes: Mice receiving high or low methyl donor diets in either the perinatal period or after weaning (eight mice per group) were tested for physical dependence on morphine. ${ }^{*} P<0.05$ for high versus low methyl donor diet. 
$(1 \mathrm{mg} / \mathrm{kg})$ dose of morphine used in these experiments in opioid-naïve mice $(P<0.05)$, as shown in Figure 3B. The dose-response relationships were equivalent after 4 days of morphine administration for the two dietary groups. Physical dependence-related jumping behavior was equal, shown in Figure 4. The jumping frequencies were 102.0 and 118.6 per 15 minutes, respectively, in the high and low methyl donor diet groups.

\section{Discussion}

Dietary factors can have significant effects on susceptibility to disease and response to treatment. Although many mechanisms are involved in these differences, long-term and even transgenerational changes in DNA methylation can be induced by exposure to diets high in fat or methyl donating compounds early in life. Epigenetic factors are known to control opioid responses under other conditions. ${ }^{16}$ In our experiments, we adopted a well established opioid response and adaptation paradigm to answer the question of whether using a dietary protocol shown by others to alter DNA methylation and inflammation we would observe changes in baseline opioid responsiveness or the propensity to develop opioid adaptations, like opioid-induced hyperalgesia, tolerance, and physical dependence. ${ }^{7}$ These dietary maneuvers, whether applied in early or later life, did not lead to gross differences in growth or development. Overall, we found only a few specific differences in opioid responses, although these may indicate possible dietary effects on pathways controlling two types of opioid responses, ie, analgesia and physical dependence. Thus, it appears that while this specific set of dietary factors may alter opioid adaptations to a limited extent, there are likely to be other environmental factors contributing as well.

Perhaps the most significant of our findings was that methyl donor supplementation of dams throughout pregnancy and continuing until weaning altered expression of physical dependence on morphine 6 weeks later. Similar dietary treatments have been linked to sustained effects on the coat color of the offspring and the morphology of the animals' tails through altered methylation of specific alleles, ${ }^{17}$ although the number of affected alleles is much larger than represented in these two examples. ${ }^{7}$ Limited information suggests a role for perinatal exposure to methyl donors in the control of higher neurological function. One example is the observation that feeding rat dams a methyl-deficient diet caused increased anxiety and enhanced learning in the offspring. ${ }^{18}$ The dietary effects of methyl donor depletion or supplementation on pain and opioid pharmacology have been largely unexplored to this point. Given the very prominent role methylation has as an epigenetic mechanism relevant to neurobiology, this may be a productive area of investigation.

It is clear that the methylation patterns of many genes related to pain, opioid analgesia, and physical dependence are controlled by DNA methylation. Most studies in this area have examined the degree of methylation of cytosine-phosphateguanine islands in the promoter regions of specific genes. These areas have been the focus of research because enhanced methylation of cytosine-phosphate-guanine islands generally leads to suppression of transcriptional activity. Examples of genes controlled by DNA methylation with established roles in opioid physical dependence are the $\mu$-opioid receptor, prodynorphin, and brain-derived neurotrophic factor genes. ${ }^{19-21}$ Despite the results of these studies showing that altered methylation can control gene expression under specific circumstances, it is important to recognize that methylation patterns vary widely between different areas of the nervous system. Thus, studies targeted to understanding methylation differences and gene expression related to specific phenotypes should be conducted in functionally relevant tissues. In the case of physical opioid dependence, a phenotype observed to be altered by dietary changes in methyl donor content, centers in the central nervous system that might be analyzed include the ventral tegmental area, locus coeruleus, and the ventral striatum, because these are brain areas related to addiction and dependence. On the other hand, our dietary data do not allow us to exclude arbitrarily other sites of opioid receptor expression, such as the spinal cord or even peripheral locations. Additional tools and approaches are available, including the possibility of comparing methylation in strains of mice developing differing degrees of dependence, using transgenic mice deficient in DNA methylation enzymes, or by the stereotactic delivery of methylation-modulating agents.

\section{Conclusion}

Dietary factors are becoming increasingly associated with altering developmental characteristics of animals, behavioral traits, and the propensity to contract specific diseases. Our work adds opioid analgesia and physical dependence to the list. Given the very diverse dietary patterns of humans, including ones involving the consumption of large amounts of fat or supplements such as folate linked to changes in DNA methylation, understanding the consequences for responses to therapeutic and recreational drugs is very important. Our findings suggest that the examination of DNA methylation patterns in specific brain regions linked to opioid analgesia 
and dependence may provide specific explanations for the dietary effects observed in this study.

\section{Acknowledgment}

This work was supported by grants from the National Institutes of Health and the Department of Veterans Affairs (R01 DA021332, I01 BX000881) to JDC at the Department of Anesthesiology, Veterans Affairs Palo Alto Health Care System, Palo Alto, CA, USA.

\section{Disclosure}

The authors report no conflicts of interest in this work.

\section{References}

1. Avena NM, Gold JA, Kroll C, Gold MS. Further developments in the neurobiology of food and addiction: update on the state of the science. Nutrition. 2012;28(4):341-343.

2. Zheng H, Zou H, Liu X, et al. Cholesterol level influences opioid signaling in cell models and analgesia in mice and humans. $J$ Lipid Res. 2012;53(6):1153-1162.

3. Frances H, Coudereau JP, Sandouk P, Clement M, Monier C, Bourre JM. Influence of a dietary alpha-linolenic acid deficiency on learning in the Morris water maze and on the effects of morphine. Eur J Pharmacol. 1996;298(3):217-225.

4. Bello NT, Patinkin ZW, Moran TH. Opioidergic consequences of dietary-induced binge eating. Physiol Behav. 2011;104(1):98-104.

5. Cohen CA, Tonkiss J, Sparber SB. Acute opiate withdrawal in rats undernourished during infancy: impact of the undernutrition method. Pharmacol Biochem Behav. 1991;39(2):329-335.

6. Vucetic Z, Kimmel J, Totoki K, Hollenbeck E, Reyes TM. Maternal high-fat diet alters methylation and gene expression of dopamine and opioid-related genes. Endocrinology. 2010;151(10):4756-4764.

7. Hollingsworth JW, Maruoka S, Boon K, et al. In utero supplementation with methyl donors enhances allergic airway disease in mice. $J$ Clin Invest. 2008;118(10):3462-3469.

8. Dominguez-Salas P, Cox SE, Prentice AM, Hennig BJ, Moore SE. Maternal nutritional status, C(1) metabolism and offspring DNA methylation: a review of current evidence in human subjects. Proc Nutr Soc. 2012;71(1):154-165.
9. Waterland RA. Do maternal methyl supplements in mice affect DNA methylation of offspring? J Nutr. 2003;133(1):238.

10. Waterland RA, Jirtle RL. Transposable elements: targets for early nutritional effects on epigenetic gene regulation. Mol Cell Biol. 2003;23(15):5293-5300.

11. Chaplan SR, Bach FW, Pogrel JW, Chung JM, Yaksh TL. Quantitative assessment of tactile allodynia in the rat paw. J Neurosci Methods. 1994;53(1):55-63.

12. Li X, Angst MS, Clark JD. A murine model of opioid-induced hyperalgesia. Brain Res Mol Brain Res. 2001;86(1-2):56-62.

13. Liang DY, Liao G, Wang J, et al. A genetic analysis of opioid-induced hyperalgesia in mice. Anesthesiology. 2006;104(5):1054-1062.

14. Berg ML, Idrees U, Ding R, Nesbit SA, Liang HK, McCarthy ML. Evaluation of the use of buprenorphine for opioid withdrawal in an emergency department. Drug Alcohol Depend. 2007;86(2-3):239-244.

15. Kest B, Palmese CA, Hopkins E, Adler M, Juni A, Mogil JS Naloxone-precipitated withdrawal jumping in 11 inbred mouse strains: evidence for common genetic mechanisms in acute and chronic morphine physical dependence. Neuroscience. 2002;115(2):463-469.

16 Liang DY, Li X, Clark JD. Epigenetic regulation of opioid-induced hyperalgesia, dependence, and tolerance in mice. J Pain. 2013;14(1): 36-47.

17. Waterland RA, Dolinoy DC, Lin JR, Smith CA, Shi X, Tahiliani KG. Maternal methyl supplements increase offspring DNA methylation at Axin Fused. Genesis. 2006;44(9):401-406.

18. Konycheva G, Dziadek MA, Ferguson LR, et al. Dietary methyl donor deficiency during pregnancy in rats shapes learning and anxiety in offspring. Nutr Res. 2011;31(10):790-804.

19. Hwang CK, Kim CS, Kim do K, Law PY, Wei LN, Loh HH. Up-regulation of the mu-opioid receptor gene is mediated through chromatin remodeling and transcriptional factors in differentiated neuronal cells. Mol Pharmacol. 2010;78(1):58-68.

20. Taqi MM, Bazov I, Watanabe H, et al. Prodynorphin CpG-SNPs associated with alcohol dependence: elevated methylation in the brain of human alcoholics. Addict Biol. 2011;16(3):499-509.

21. Fuchikami M, Morinobu S, Segawa M, et al. DNA methylation profiles of the brain-derived neurotrophic factor (BDNF) gene as a potent diagnostic biomarker in major depression. PloS One. 2011;6(8): e23881.
Journal of Pain Research

\section{Publish your work in this journal}

The Journal of Pain Research is an international, peer-reviewed, open access, online journal that welcomes laboratory and clinical findings in the fields of pain research and the prevention and management of pain. Original research, reviews, symposium reports, hypothesis formation and commentaries are all considered for publication.

\section{Dovepress}

The manuscript management system is completely online and includes a very quick and fair peer-review system, which is all easy to use. Visit http://www.dovepress.com/testimonials.php to read real quotes from published authors. 